\title{
CALCULATIONS OF POINT-DEFECT PROPERTIES IN COPPER, SILVER, AND GOLD BASED ON THREE-BODY INTERACTIONS
}

\author{
R. BAUER ${ }^{a}$, W. MAYSENHÖLDER ${ }^{a, b}$ and A. SEEGER $a, b$ \\ a Universität Stuttgart, Institut für Theoretische und Angewandte Physik, Pfaffenwaldring 57, \\ D 7000 Stuttgart 80, Fed. Rep. Germany \\ b Max-Planck-Institut für Metallforschung, Institut für Physik, Heisenbergstr. 1, D 7000 Stuttgart 80, Fed. Rep. Germany
}

Received 13 April 1982

\begin{abstract}
Long-term trends in the calculation of defect properties in the noble metals are criticezed. On physical grounds it is argued that the model potentials used should allow for many-body interactions. Results based on potentials which include threebody interaction terms are presented for $\mathrm{Cu}, \mathrm{Ag}$, and $\mathrm{Au}$.
\end{abstract}

The last twenty years have seen many attempts to calculate formation energies and formation volumes of vacancies and interstitials in face-centred cubic metals. (Johnson [1] has given an authoritative review; for recent developments see refs. [2,3].) Judged by the effort spent, however, the progress achieved must be considered small. The majority of the model calculations on point-defects in $\mathrm{Cu}$ carried out during the above-mentioned period suffer from the following principal deficiencies: (i) The model potential is fitted either to the cohesive energy and then yields unrealistic values for the defect formation energies (see, e.g. ref. [4]), or to the vacancy formation energy, a quantity which one would wish to calculate [2-4]. (ii) Only two-body potentials have been used. This implies the validity of Cauchy's relation $c_{12}=c_{44}$ between the elastic moduli unless the pair potential is non-central (i.e., angle-dependent) or an external pressure $p=\left(c_{12}-c_{44}\right) / 2$, called the Cauchy pressure, is applied. In $\mathrm{Cu}$ and even more so in $\mathrm{Ag}$ and $\mathrm{Au}$ Cauchy's relation is violated; hence an exact matching of the elastic moduli by the usual two-body potentials is impossible. Both ways of accounting for the deviations from Cauchy's relation in $\mathrm{Cu}, \mathrm{Ag}$, and $\mathrm{Au}$ with pair potentials have been tried [5-7] but did not prove successful. (iii) The experimental value of the vacancy relaxation volume in $\mathrm{Cu}[-(0.20 \pm 0.05) \Omega$ [8], $\Omega=$ atomic volume] could not be reproduced ${ }^{\ddagger 1}$.
The situation can be drastically improved with the aid of model potentials developed recently [10]. The basic idea is to expand the cohesive energy per atom $E_{\mathrm{c}}$ in $m$-body contributions $E_{\mathrm{c}}^{(m)}$ (cf. ref. [11]) according to

$E_{\mathrm{c}}\left(\left\{\boldsymbol{R}_{i}\right\}\right)=\sum_{m=1}^{M} E_{\mathrm{c}}^{(m)} \quad(i=1 \ldots N ; \quad M \leqslant N)$,

with

$E_{\mathrm{c}}^{(m)}=\frac{1}{m !} \sum_{g_{2}=2}^{N} \ldots \sum_{g_{m}=2}^{N} E^{(m)}\left(R_{1}, R_{g_{2}}, \ldots, R_{g_{m}}\right)$,

where $N$ denotes the total number of atoms in the crystal and $\boldsymbol{R}_{\boldsymbol{i}}$ their positions. Primes indicate the exclusion of terms with $g_{j}=g_{k}$. It is understood that the $m$-body contribution $E_{c}^{(m)}$ cannot be decomposed into a sum of contributions of order lower than $m$. In spite of the formal appearance of (1) the following conclusions pertaining to vacancy formation can be drawn from (1) in a straightforward manner.

The vacancy formation energy in a rigid lattice $E_{1 \mathrm{~V}, 0}^{\mathrm{F}}$, which differs only little from the true vacancy

\#1 The early calculations of Seeger and Mann [9] giving values between $-0.1 \Omega$ and $-0.3 \Omega$, are an exception in this respect as a consequence of the application of Born's lattice thcory with non-central forces. 
formation energy $E_{1 \mathrm{~V}}^{\mathrm{F}}$, can be written as the sum of a separation energy $E_{\text {sep }}$ - the atom is removed from the crystal interior to infinity - and an addition energy $E_{\text {add }}$ - the atom is put back to a surface kink position of the crystal:

$E_{1 \mathrm{~V}, 0}^{\mathrm{F}}=E_{\text {sep }}+E_{\mathrm{add}}$.

From (1) follow

$E_{\mathrm{sep}}=-\sum_{m=1}^{M} m E_{\mathrm{c}}^{(m)}, \quad E_{\mathrm{add}}=\sum_{m=1}^{M} E_{\mathrm{c}}^{(m)}=E_{\mathrm{c}}$,

hence

$$
\begin{aligned}
& E_{1 \vee, 0}^{\mathrm{F}}=-\sum_{m=2}^{M}(m-1) E_{\mathrm{c}}^{(m)} \\
& \quad=-\left(E_{\mathrm{c}}-E_{\mathrm{c}}^{(1)}\right)-\sum_{m=3}^{M}(m-2) E_{\mathrm{c}}^{(m)} .
\end{aligned}
$$

If contributions with $m \geqslant 3$ are neglected the vacancy formation energy in a rigid lattice is equal to the difference between the cohesive energy and the structureindependent one-body contribution $E_{\mathrm{c}}^{(1)}=E^{(1)}\left(R_{1}\right)$. The main physical origin of $E_{\mathrm{c}}^{(1)}$ is the delocalization of the valence electrons. In $\mathrm{Cu}, \mathrm{Ag}, \mathrm{Au}$ this contribution is of the order of magnitude of $\mathrm{eV}$ and must therefore not be neglected when constructing a model potential which fits the cohesive energy $E_{\mathrm{c}}\left(E_{\mathrm{c}}^{(1)}\right.$ is contained in $E_{\mathrm{c}}$, but not in $E_{1 \mathrm{~V}}^{\mathrm{F}}$ !) ${ }^{\ddagger 2}$.

The derivative of (5) with respect to the atomic volume,

$\frac{\partial E_{1 \mathrm{~V}, 0}^{\mathrm{F}}}{\partial \Omega}=\frac{\partial E_{\mathrm{c}}^{(1)}}{\partial \Omega}-\sum_{m=3}^{M}(m-2) \frac{\partial E_{\mathrm{c}}^{(m)}}{\partial \Omega}$,

is a measure of the tendency of the vacancy to relax (cf. ref. [14]). For $\mathrm{Cu}, \mathrm{Ag}, \mathrm{Au}$ the first term on the right-hand side is approximately zero. Hence, as long as we consider two-body contributions $E_{\mathrm{c}}^{(2)}$ only, we expect virtually no vacancy relaxation. In agreement with this, pair-potential calculations usually produce very small vacancy relaxation volumes (around -0.02 $\Omega$ ), which come mainly from the derivative of the

\$2 The contribution $E^{(3)}$ has been considered the first time by Foreman and Lidiard [12], contributions $E^{(m)}$ with $m>3$ by Burton [13]. vacancy relaxation energy not contained in (6). In view of the experimental value of about $-0.2 \Omega$ we must conclude that many-body interactions do indeed play an important rôle in the noble metals.

In order to keep the computations within reasonable bounds, we neglect in the following $m$-body contributions with $m \geqslant 4$. The two- and three-body interactions are taken to be

$$
\begin{aligned}
& E^{(2)}\left(r_{i j}\right)=A_{\mathrm{BM}} \exp \left[-r_{i j} / \rho\right]-A_{\mathrm{vdW}} / r_{i j}^{n} \mathrm{vdW}, \\
& E^{(3)}\left(r_{i j}, r_{j k}, r_{k i}\right)=A_{\mathrm{AT}} \frac{1+3 \cos \alpha \cos \beta \cos \gamma}{\left(r_{i j} r_{j k} r_{k i}\right)^{n_{\mathrm{AT}}}},
\end{aligned}
$$

where $r_{i j}=\left|R_{i}-R_{j}\right|$ and $\alpha, \beta, \gamma$ are the angles in the triangle $\left\{\boldsymbol{R}_{i}, \boldsymbol{R}_{j}, \boldsymbol{R}_{k}\right\}$. The pair contribution $E^{(2)}$ consists of a Born-Mayer repulsion and a van der Waals attraction with the exponent (usually $n_{\mathrm{vdW}}=6$ ) kept variable. $E^{(3)}$ has the form of an Axilrod-Teller potential [15], again with a variable exponent (usually $n_{\text {AT }}=3$ ). The physical picture behind these formulae is the interaction between neutral polarizable bodies. The conduction electrons do not enter the equations explicitly. Their main rôle is to compensate the ion charge within a Wigner-Seitz cell. In addition they contribute to $E_{\mathrm{c}}^{(1)}$ and screen the dipolar interactions in (7) and (8) at large distances. In the interest of simplicity this screening is taken into account by means of cut-off radii

$a_{\mathrm{NN}}=1.75 a_{0} / 2, \quad a_{2 \mathrm{~N}}=2.10 a_{0} / 2, \quad a_{3 \mathrm{~N}}=2.65 a_{0} / 2$,

$\left(a_{0}=\right.$ elementary cube edge) separating the nearest neighbours (NN) from the next-nearest neighbours $(2 \mathrm{~N})$, the $2 \mathrm{~N}$ from the $3 \mathrm{~N}$, or the $3 \mathrm{~N}$ from the more distant neighbours in the perfect lattice. The numerical values in (9) are chosen such that when applied to an interstitial site, a similar number of atoms are cut off as for a lattice site. This is reasonable since the local relaxation volume for an interstitial is about one atomic volume, hence the density of atoms around an interstitial about the same as in the perfect lattice.

In our model the Born-Mayer repulsion is restricted to nearest neighbours only $\left(a_{\mathrm{BM}}=a_{\mathrm{NN}}\right)$, the van der Waals attraction extends to $2 \mathrm{~N}$ or sometimes to $3 \mathrm{~N}\left(a_{\mathrm{vdW}}=a_{2 \mathrm{~N}}\right.$ or $\left.a_{3 \mathrm{~N}}\right)$, whereas the AxilrodTeller interaction includes the $3 \mathrm{~N}\left(a_{\mathrm{AT}}=a_{3 \mathrm{~N}}\right)$.

An ideal model potential should reproduce all properties of the perfect crystal. By the choice of $A_{\mathrm{BM}}, \rho$, 
Table 1

Input and model parameters.

\begin{tabular}{lccc}
\hline & $\mathrm{Cu}$ & $\mathrm{Ag}$ & $\mathrm{Au}$ \\
\hline$a_{0}\left[10^{-10} \mathrm{~m}\right]$ & 3.592 & 4.063 & 4.061 \\
$c_{11}\left[10^{11} \mathrm{~N} \mathrm{~m}^{-2}\right]$ & 1.810 & 1.345 & 2.031 \\
$c_{12}\left[10^{11} \mathrm{~N} \mathrm{~m}^{-2}\right]$ & 1.265 & 0.995 & 1.707 \\
$c_{44}\left[10^{11} \mathrm{~N} \mathrm{~m}^{-2}\right]$ & 0.840 & 0.522 & 0.457 \\
$E_{\mathrm{c}}[\mathrm{eV}]$ & -3.507 & -2.960 & -3.802 \\
$E_{\mathrm{c}}^{(1)}[\mathrm{eV}]$ & -1.924 & -1.169 & -1.613 \\
$a_{\mathrm{vdW}}$ & $a_{2 \mathrm{~N}}$ & $a_{3 \mathrm{~N}}$ & $a_{3 \mathrm{~N}}$ \\
$n_{\mathrm{AT}}$ & 3 & 3 & 4 \\
\hline
\end{tabular}

$A_{\mathrm{vdW}}$, and $A_{\mathrm{AT}}$ we have matched exactly the lattice constant $a_{0}$ and the elastic moduli $c_{11}, c_{12}, c_{44}$ (table 1 ; all values extrapolated to absolute zero, because we do not consider thermal vibrations in the calculations), by the choice of $E_{\mathrm{c}}^{(1)}$ the cohesive energy $E_{\mathrm{c}}$. The parameters $a_{\mathrm{vdW}}$ and $n_{\mathrm{AT}}$ were selected with the phonon frequencies in mind. In this respect the outcome is satisfactory at least for $\mathrm{Cu}$ and $\mathrm{Ag}$. The remaining parameter $n_{\mathrm{vdW}}$ has very little influence on the phonon frequencies but a strong one on the formation energies. The physical picture outlined above requires the van der Waals exponent to be 6 or close to this value. The results of table 2 have been obtained with $n_{\mathrm{vdW}}=6$.

Table 2

Formation energies $E^{\mathrm{F}}$ and formation volumes $V \mathrm{~F}$ of vacancies and interstitials, and divacancy binding energies $E^{\mathrm{B}}$ in $\mathrm{Cu}, \mathrm{Ag}, \mathrm{Au}$ obtained with model potential including threebody interactions $\left(n_{\mathrm{vdW}}=6\right)$.

\begin{tabular}{|c|c|c|c|}
\hline & $\mathrm{Cu}$ & $\mathrm{Ag}$ & $\mathbf{A u}$ \\
\hline \multicolumn{4}{|l|}{$E^{\mathrm{F}}[\mathrm{eV}]$} \\
\hline $\begin{array}{l}\text { monovacancy } \\
\text { interstitials }\end{array}$ & 1.05 & 0.90 & 0.85 \\
\hline$(100\rangle$-split & 2.48 & 2.57 & 2.26 \\
\hline octahedral & 3.07 & 2.72 & 2.29 \\
\hline$\langle 101\rangle$-split & 2.97 & 2.66 & 2.19 \\
\hline (101)-crowdion & 2.91 & 2.55 & 2.13 \\
\hline$E_{2 \mathrm{~V}}^{\mathrm{B}}[\mathrm{eV}]$ & 0.11 & 0.03 & -0.04 \\
\hline \multicolumn{4}{|l|}{$V^{\mathrm{F}_{[\Omega]}}$} \\
\hline $\begin{array}{l}\text { monovacancy } \\
\text { interstitials }\end{array}$ & 0.79 & 0.69 & 0.63 \\
\hline$\langle 100\rangle$-split & 1.12 & 0.97 & 0.97 \\
\hline octahedral & 1.34 & 1.22 & 1.19 \\
\hline (101)-split & 1.14 & 1.04 & 0.55 \\
\hline (101)-crowdion & 1.14 & 0.98 & 0.43 \\
\hline
\end{tabular}

Because of the current controversies surrounding the $E^{F}$ values of vacancies in the noble metals we have varied $n_{\mathrm{vdW}}$ by \pm 1 and shall report below a result for $n_{\mathrm{vdW}}=5$.

We took advantage of the defect symmetry by adopting the model of Seeger and Mann $[6,16]$. Lowsymmetry configurations $(\langle 111\rangle$-split and tetrahedral interstitials) could not be treated with the available computing capacity $\neq 3$.

The results for formation energies $E^{\mathrm{F}}$ and formation volumes $V^{\mathrm{F}}$ of vacancies and of four interstitial configurations as well as for the divacancy binding energies are shown in table 2. Almost all quantities listed decrease when one passes from $\mathrm{Cu}$ to $\mathrm{Au}$. This is due to the increasing weight of the three-body interaction reflecting the increasing violation of $c_{12}=c_{44}$ when going from $\mathrm{Cu}$ to $\mathrm{Au}$. The exceptions originate from the different ranges of the van der Waals attraction. For $a_{\mathrm{vdW}}=a_{2 \mathrm{~N}}(\mathrm{Cu})$ the formation energy of the $\langle 100\rangle$-split interstitial is distinctly lower than that of the other interstitials, whereas for $a_{\mathrm{vdW}}=a_{3 \mathrm{~N}}(\mathrm{Ag}$ and $\mathrm{Au}$ ) the four interstitial configurations are rather close together. While a larger range of the van der Waals attraction in $\mathrm{Ag}$ and $\mathrm{Au}$ is plausible, in view of certain inadequacies in the potentials for $\mathrm{Ag}$ and $\mathrm{Au}$ (in the case of $\mathrm{Au}$ the Einstein frequency comes out too low) the sequences of interstitial energies in $\mathrm{Ag}$ and $\mathrm{Au}$ as well as the negative divacancy binding energy in $\mathrm{Au}$ should be considered with caution.

As indicated above, the absolute values of $E^{\mathrm{F}}$ are sensitive to modifications of the exponent $n_{\mathrm{vdW}}$. E.g., we would get formation energies of $1.32 \mathrm{eV}$ or 2.13 $\mathrm{eV}$ for a vacancy or a $\langle 100\rangle$-split in $\mathrm{Cu}$ if we would use $n_{\mathrm{vd} \mathrm{W}}=5$. (Note that the formation.energy of a Frenkel pair of about $3.5 \mathrm{eV}$ remains virtually unchanged.)

The interstitial formation volumes appear to be too high (cf. refs. $[8,3]$ ). This is presumably due to the pressure derivative of the bulk modulus which turns out to be too large compared with the experimental values. On the other hand, the vacancy relaxation volumes come out reasonable; in the case of $\mathrm{Cu}$ we ob-

\footnotetext{
\#3 For a comprehensive description of the calculations see ref. [17]. In [17] the reader may note a discrepancy of $1 \mathrm{eV}$ in the $\mathrm{Au}$ interstitial formation energies in the table on p. 123 and the diagram on p. 124; the former values are the correct ones.
} 
tain very good agreement with experiment.' Divacancy binding volumes are negligible.

Similar formation energies may be obtained for $\mathrm{Cu}$ (but not for $\mathrm{Ag}$ and $\mathrm{Au}$ ) with a pure pair potential $\left(A_{\mathrm{AT}}=0\right.$ ) fitted to the shear moduli (not to the bulk modulus as usual). However, the formation volumes (e.g., $V_{1 V}^{\mathrm{F}}=0.94 \Omega$ ) obtained in this way are far too large. This result supports our general philosophy of the rôle of three-body interactions in the noble metals.

Complete stability investigations require the consideration of many low-symmetry configurations and were therefore impossible. In view of the controversial question of the metastability of the crowdion we have performed simple stability tests, in which the crowdion or the crowdion and its two nearest neighbours were moved by small amounts from their equilibrium positions in an otherwise rigid lattice. In no case was a decrease of energy connected with such a shift, i.e. no obvious instability could be detected.

The authors acknowledge the collaboration of Dr. R.K. Leutz at the beginning of the computations.

\section{References}

[1] R.A. Johnson, J. Phys. F3 (1973) 295.
[2] P.H. Dederichs, C. Lehmann, H.R. Schober, A. Scholz and R. Zeller, J. Nucl. Mater. $69+70$ (1978) 176.

[3] K.W. Ingle, R.C. Perrin and H.R. Schober, J. Phys. F11 (1981) 1161.

[4] M. Doyama and R.M.J. Cotterill, Lattice defects and their interactions, ed. R.R. Hasiguti (Gordon and Breach, New York, 1967).

[5] K.M. Miller and P.T. Heald, Phys. Stat. Sol. (b) 67 (1975) 569.

[6] K.M. Miller and P.T. Heald, Phys. Stat. Sol. (b) 78 (1976) 341.

[7] R.A. Johnson and W.D. Wilson, Interatomic potentials and simulation of lattice defects, eds. P.C. Gehlen, J.R. Beeler and R.I. Jaffee (Plenum Press, New York, 1972) p. 301.

[8] H.-G. Haubold and D. Martinsen, J. Nucl. Mater. 69 +70 (1978) 644 .

[9] A. Seeger and E. Mann, J. Phys. Chem. Solids 12 (1960) 326.

[10] R. Bauer, to be published.

[11] J.W. Martin, J. Phys. C8 (1975) 2837.

[12] A.J.E. Foreman and A.B. Lidiard, Philos. Mag. 8 (1963) 97.

[13] J.J. Burton, Phys. Rev. 182 (1969) 885.

[14] A.J.E. Foreman, Philos. Mag. 8 (1963) 1211.

[15] B.M. Axilrod and E. Teller, J. Chem. Phys. 11 (1943) 299.

[16] A. Seeger, E. Mann and R.V. Jan, J. Phys. Chem. Solids 23 (1962) 639.

[17] W. Maysenhölder, Dissertation Stuttgart (1981). 